\title{
Evaluación de manifestaciones patológicas en edificios en función de la orientación geográfica
}

\author{
W. Mazer ${ }^{1}$, L. M. R. Silva², E. Lucas ${ }^{3}$, F. C. M. Santos ${ }^{4}$. \\ ${ }^{1}$ Professor do Departamento Acadêmico de Construcción Civil da UTFPR - Campus Curitiba. \\ ${ }^{2}$ Arquiteta da Estruktor Patologia das Construcciones. \\ 3 Tecnóloga em Concreto. \\ ${ }^{4}$ Tecnóloga em Concreto da Estruktor Patologia das Construcciones.
}

\section{Información del artículo \\ DOI: \\ http://dx.doi.org/10.21041/ra. \\ v6i2.135 \\ Artículo recibido el 16 de diciembre de 2015, revisado bajo las políticas de publicación de la Revista ALCONPAT y aceptado el 12 de abril de 2016. Cualquier discusión, incluyendo la réplica de los autores, se publicará en el primer número del año 2017 siempre y cuando la información se reciba antes del cierre del tercer número del año 2016.}

C 2016 ALCONPAT Internacional

Información Legal

Revista ALCONPAT, Año 6, №. 2,

Mayo - Agosto 2016,, es una

publicación cuatrimestral de la

Asociación Latinoamericana de Control de Calidad, Patología y Recuperación de la Construcción, Internacional, A.C. $\mathrm{Km}$. 6, antigua carretera a Progreso, Mérida Yucatán, C.P. 97310,

Tel.5219997385893,

Tel.5219997385893,
alconpat.int@gmail.com, Página Web: www.revistaalconpat.org

Editor responsable: Dr. Pedro Castro Borges. Reserva de derechos al uso exclusivo No.04-2013-011717330300203, eISSN 2007-6835, ambos otorgados por el Instituto Nacional de Derecho de Autor. Responsable de la Derecho de Autor. Responsable de la última actualización de este número, Unidad de Informática ALCONPAT, Ing. Elizabeth Sabido Maldonado, Km. 6, antigua carretera a Progreso, Mérida Yucatán, C.P. 97310, fecha de última modificación: 30 de mayo de 2016.

Las opiniones expresadas por los autores no necesariamente reflejan la postura del editor.

editor.

Queda totalmente prohibida la
reproducción total o parcial de los

contenidos e imágenes de la publicación sin previa autorización de la

ALCONPAT Internacional A.C.

\section{RESUMEN}

Con el propósito de evaluar la incidencia de manifestaciones patológicas en fachadas de edificios, correlacionadas con la dirección geográfica, se analizaron en este estudio 52 edificios de uso residencial y comercial distribuidos en varias partes de la ciudad de Curitiba - Brasil. Se observó que todos los edificios evaluados tuvieron algún tipo de manifestación patológica, y 25 de éstas en las fachadas. Se encontró que las manifestaciones patológicas en fachadas aparecen en diferentes cantidades y direcciones, habiéndose identificado una relación con la variación de la temperatura y la dirección del viento en función de la orientación geográfica.

Palabras Claves: durabilidad; fachada; dirección geográfica; manifestación patológica.

\section{ABSTRACT}

In order to evaluate the incidence of pathological manifestations in buildings facades, correlating with the geographic directions, this study analyzed 52 residential and commercial buildings distributed in various parts of the city of Curitiba - Brazil. It was observed that all the buildings surveyed had some kind of pathological manifestation, and 25 had problems on the facades. It was found that the pathological manifestations in facades appear in different amounts in different directions, having identified a relationship with temperature variation and the direction of the wind, according to geographical direction.

Keywords: durability; facade; geographic direction; pathological manifestation.

\section{RESUMO}

Com a finalidade de avaliar a incidência de manifestações patológicas em fachadas de edifícios, correlacionando com as direções geográficas, neste trabalho foram analisados 52 edifícios de uso residencial e comercial distribuídos em diversas regiões da cidade de Curitiba - Brasil. Foi observado que todos os edifícios vistoriados apresentaram algum tipo de manifestação patológica, sendo que 25 apresentaram problemas nas fachadas. Verificou-se que as manifestações patológicas nas fachadas aparecem em quantidades diferentes nas diferentes direções, tendo sido identificada uma relação com a variação de temperatura e com a direção dos ventos, conforme a direção geográfica.

Palavras claves: durabilidade; fachadas; direção geográfica; manifestações patológicas.

Autor a contactar: Wellington Mazer (wmazer@utfpr.edu.br) 
Revista ALCONPAT, Volumen 6, Número 2, Mayo - Agosto 2016, Páginas 145 - 156

\section{INTRODUCCIÓN}

La construcción civil convive con la ocurrencia de manifestaciones patológicas es sus obras, originado por los más variados motivos. Según el método de Evaluación determinado por la ASTM E632-82 (1996), que analiza la durabilidad de las construcciones, existen diversos factores que influyen en las edificaciones y pueden ser separados en cinco diferentes grupos, de acuerdo con su naturaleza: factores atmosféricos, biológicos, de carga, de incompatibilidad y de uso, según se ve en la Figura 1.

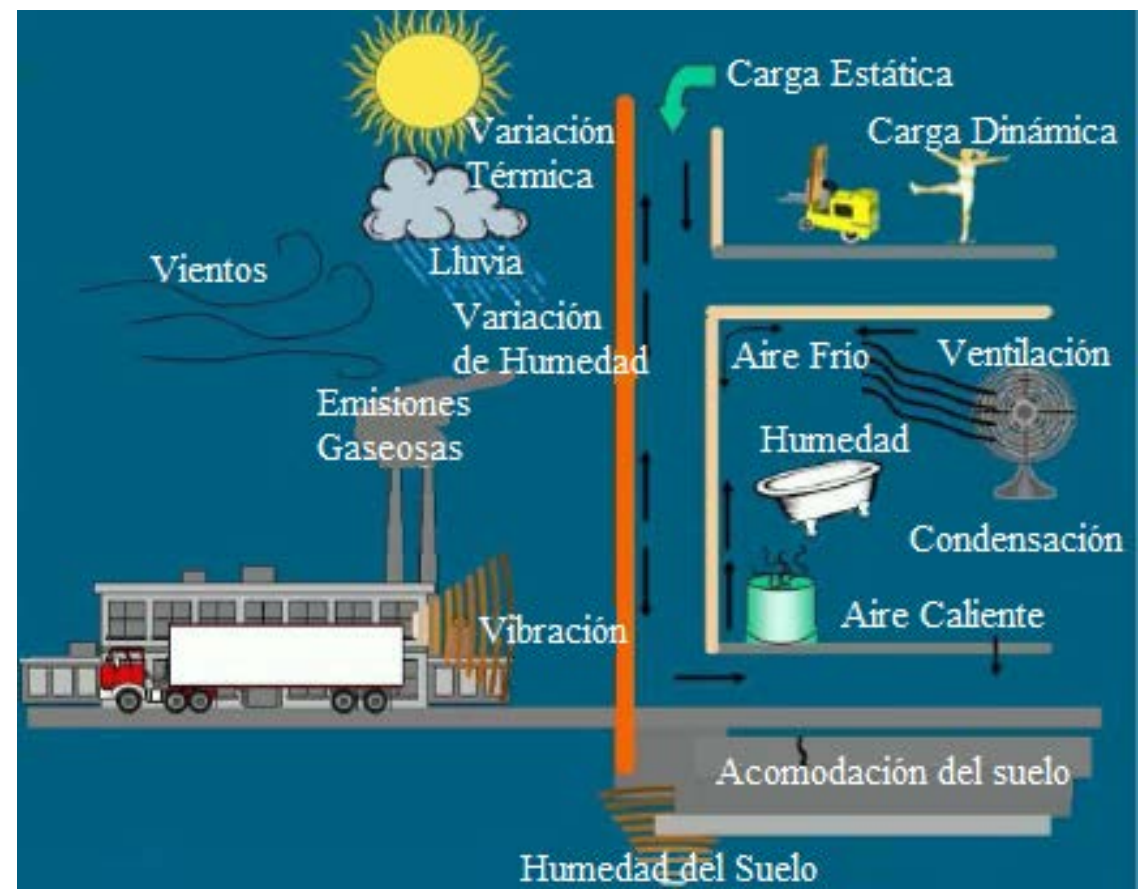

Figura 1. Factores de degradación actuantes sobre fachadas (Fuente: Antunes; 2010).

Los factores atmosféricos son definidos como los grupos de factores asociados al medio ambiente, que incluyen la temperatura, radiación solar, contaminantes del aire y la humedad proveniente de la lluvia y nieve o hielo. Tales factores siempre actúan sobre las edificaciones y pueden tener una serie de variaciones, en función de las estaciones del año y la localización geográfica del desarrollo. La variación de la temperatura puede producir una variación dimensional del material (dilatación o contracción) (Resende, Barros y Campos, 2001).

Los agentes biológicos son caracterizados principalmente por hongos o pequeñas especies de vegetaciones que necesitan de condiciones especiales para desarrollarse, tales como: temperaturas entre $10^{\circ} \mathrm{C} \mathrm{y} 35^{\circ} \mathrm{C}$, humedad relativa elevada, presencia de nitrógeno, fósforo, carbono y en menor cantidad hierro, calcio, cloro, magnesio, entre otros componentes químicos (Shirakawa, 1995). Lo que demuestra que la presencia de agua, en cualquier estado, es fundamental para el desarrollo de estos organismos y coloca las fachadas de edificios como uno de los locales más propicios para su aparición.

Los factores de carga pueden ser representados por la acción física de las lluvias y los vientos sobre la fachada de los edificios. Los factores de incompatibilidad pueden ocurrir debido a algún tipo de divergencia química o física; la química puede ocurrir debido a diferencias en la composición química de materiales de base y revestimiento o adición incorrecta de algún material como el yeso en el mortero y la física cuando un revestimiento proporciona cargas insustentables la base o sustrato, generando desplazamientos. 
Revista ALCONPAT, Volumen 6, Número 2, Mayo - Agosto 2016, Páginas 145 - 156

Finalmente, los factores de uso son determinados por la influencia directa de los usuarios de la edificación sobre los materiales componentes de la edificación, en toda la vida útil de la misma el proyecto, ejecución, uso, operación y mantenimiento. (Resende, Barros y Campos, 2001).

Según Barros y Sabbatini (2001), los problemas patológicos más frecuentes son: formación de manchas de humedad, con desarrollo de moho; astillamiento y desplazamientos entre capas de friso y el yeso y formación de fisuras y grietas.

Uno de los factores ampliamente estudiados es la acción atmosférica sobre las fachadas. Romero et al (2011) estudiaron la Torre Colpatria, en Colombia, y observaron la existencia de fisuración, carbonatación y ataque por sulfatos en las fachadas del edificio en estudio, relacionando los daños observados con factores como la altura en que ocurrió el daño, la orientación geográfica de la fachada y la dirección predominante de los vientos y lluvias. Lembo (2011) estudió tres edificios de la región de la Basilicata donde verificó la influencia del relieve alrededor de la construcción y de la dirección predominante de los vientos, habiendo constatado, principalmente, problemas de infiltración y ventilación.

Melo y Carasek (2014) verificaron la existencia de una relación entre la dirección predominante de las lluvias y manchas existentes en fachadas de edificios históricos. Los autores también verificaron la existencia de manchas debido a la acción microbiológica, influenciada por la radiación solar.

Entre las diversas manifestaciones patológicas encontradas en las fachadas de edificios, Galletto y Andrello (2013) observaron que la principal incidencia son las fisuras, seguido de las manchas y separaciones de los revestimientos cerámicos, tanto en edificios nuevos como antiguos. Los autores creen que las fisuras existentes en los sistemas de juntas, provenientes de variaciones térmicas y de humedad, pueden haber influenciado en la ocurrencia de los demás daños. Estos mismos daños fueron observados por Abreu et al (2013) que estudiaron fachadas revestidas con elementos cerámicos y con pinturas.

Los astillamientos de los revestimientos fueron estudiados por Japiassú et al (2014) que analizaron la interface entre mortero y cerámicas en fachadas de edificios ejecutados en los siglos XIX y XX, donde se constató que la adherencia entre estos materiales, influye directamente en estos astillamientos, teniendo una gran relación el diámetro de los agregados y las piezas cerámicas.

Diversos autores (Melo y Carasek, 2014; Galletto y Andrello, 2013) verificaron la influencia de algunos parámetros ambientales como radiación solar, dirección de las lluvias, variación térmica y humedad en la incidencia de manifestaciones patológicas, siendo que estos factores pueden variar de intensidad según la dirección geográfica de la fachada de un edificio, generando una mayor incidencia de daños en alguna de las fachadas.

En este contexto el presente trabajo planteó como objetivos verificar cuales son las principales manifestaciones patológicas incidentes en fachadas de edificios residenciales y comerciales en Curitiba/Paraná y las correlaciona con la dirección geográfica en que se encuentran.

\section{PROCEDIMIENTOS METODOLÓGICOS}

El desarrollo de esta investigación ocurrió en la ciudad de Curitiba, situada en la primera meseta del estado de Paraná, a una altitud aproximada de 950 m y a 100 km de distancia del litoral, siendo dividida en dos etapas, la primera constituida de una muestra de 52 edificios de la ciudad con localizaciones indicadas en la Figura 2, con edades entre 1 y 50 años de construcción. Las alturas de los edificios variaron de 4 pisos a 23 pisos, siendo que 31 edificios poseen 1 sótano, 5 poseen 2 sótanos y los otros 16 no tienen sótano. Los edificios se localizan, en su gran mayoría, próximos a la región central de la ciudad y en urbanizaciones donde existe una gran concentración de edificios.

Evaluación de manifestaciones patológicas en edificios en función de la orientación... 
Revista ALCONPAT, Volumen 6, Número 2, Mayo - Agosto 2016, Páginas 145 - 156

La Evaluación de todos los edificios utilizados en esta investigación se debió a la procura de una empresa especializada en la elaboración de informes de inspección. En esta etapa fueron identificadas las principales manifestaciones patológicas existentes en los edificios. Las inspecciones fueron efectuadas en áreas comunes de los edificios, en particular los sótanos, planta baja, tanques de agua, azoteas y en las cuatro fachadas de cada edificio. En las áreas comunes fueron realizadas inspecciones visuales con registros fotográficos y mediciones de espesores de fisuras y en las fachadas fue hecha una inspección visual, utilizando técnicas de tanteo de las regiones críticas usando fundas de seguridad o eslingas para recorrer la fachada, detectando fisuras, desplazamientos de revestimientos, manchas de eflorescencia y filtraciones.

\section{Curitiba}

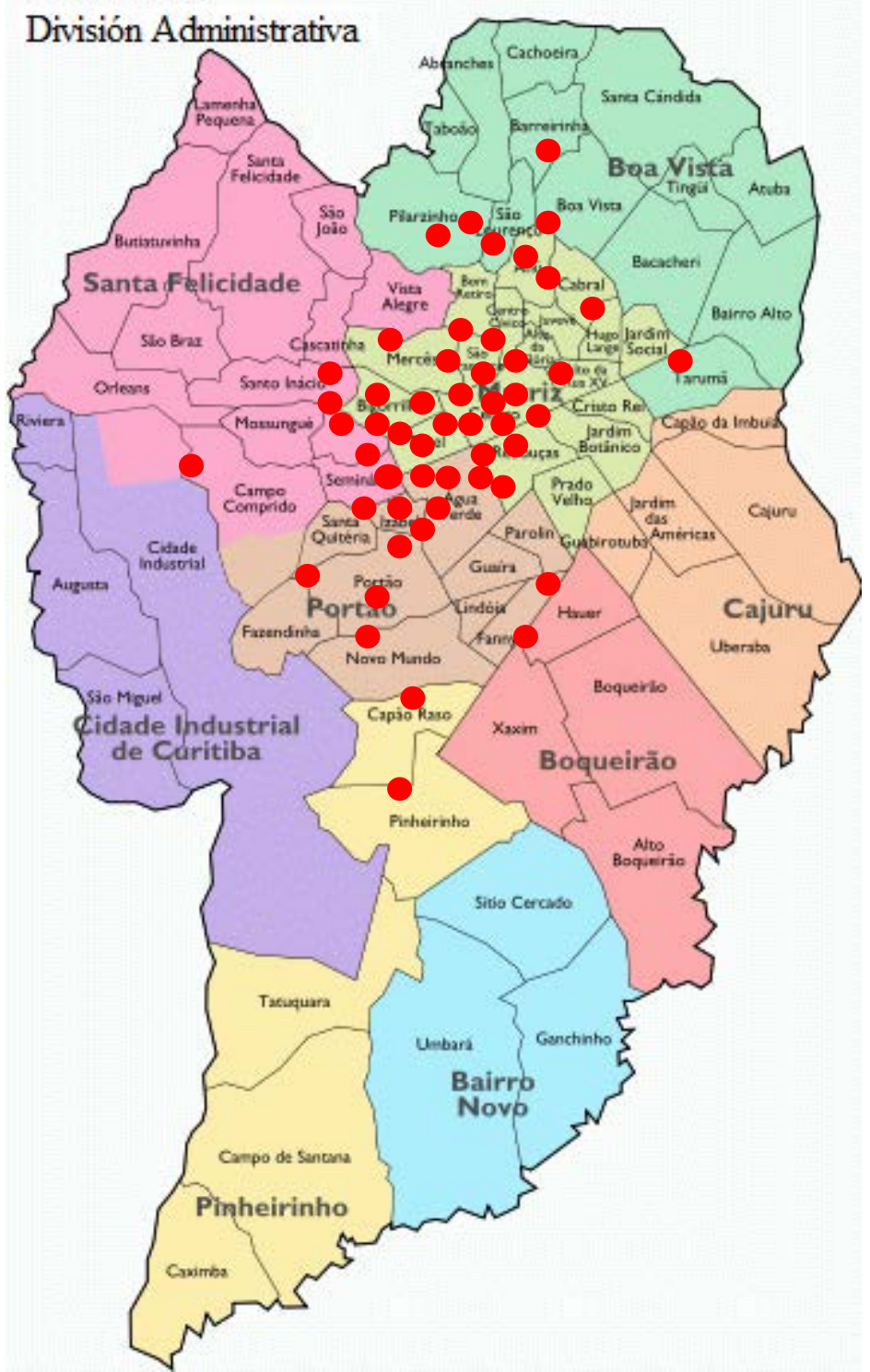

Figura 2. Localización de los edificios inspeccionados.

En una segunda etapa, fueron inspeccionadas las 25 fachadas de edificios de la muestra inicial que presentaron manifestaciones patológicas, relacionando las fachadas de los edificios con la posición geográfica para verificar si existe alguna relación entre las manifestaciones patológicas y la posición geográfica. Esos 25 edificios tienen alturas variando de 9 a 22 pisos. Los edificios que no 
Revista ALCONPAT, Volumen 6, Número 2, Mayo - Agosto 2016, Páginas 145 - 156

tienen problemas en las fachadas fueron descartados en esta etapa. En la Figura 3 están indicadas las regiones utilizadas en la segunda etapa de la investigación, donde están situadas los edificios que si tienen fallas en las fachadas.

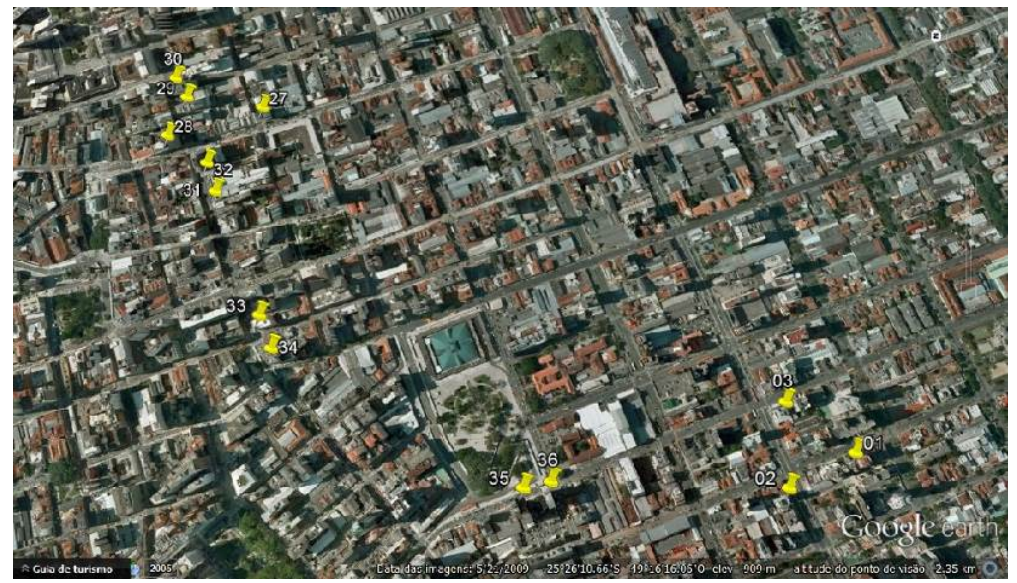

a) Región central de la ciudad.

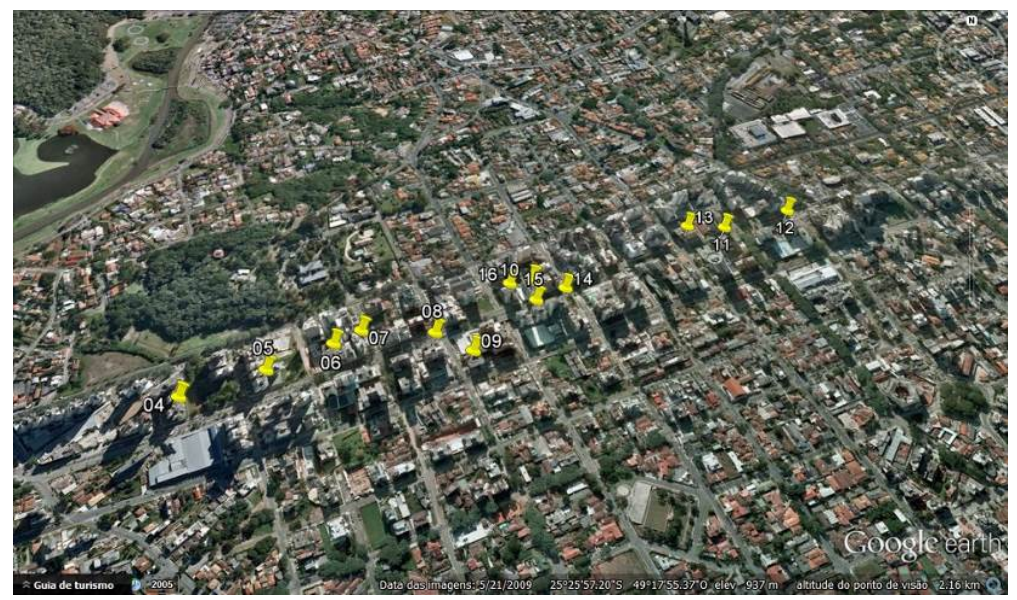

b) Región de las urbanizaciones Bigorrilho y Mercês.

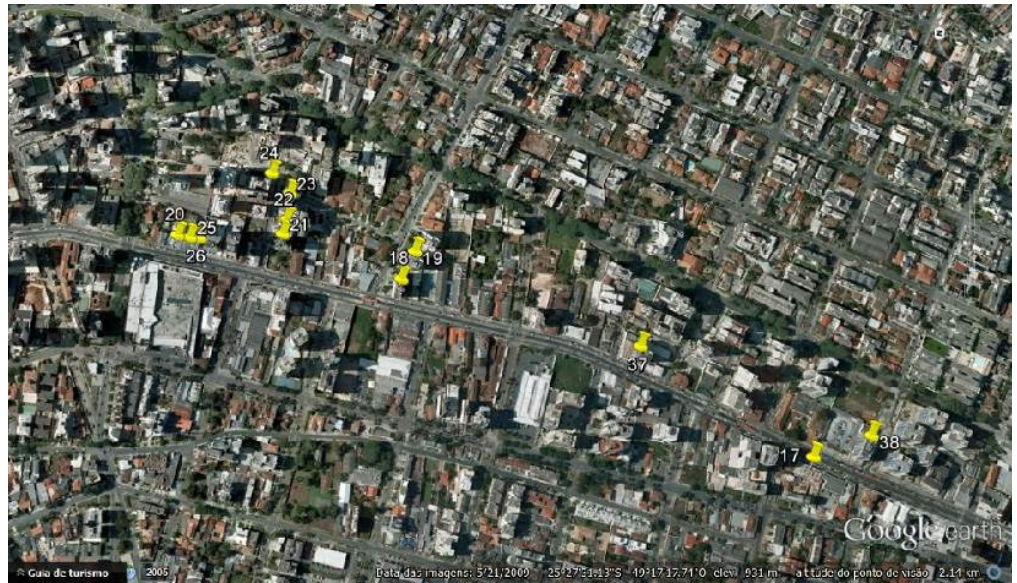

c) Región de la urbanización Água Verde.

Figura 3. Localización de las regiones de la segunda etapa del estudio.

Evaluación de manifestaciones patológicas en edificios en función de la orientación... 
Revista ALCONPAT, Volumen 6, Número 2, Mayo - Agosto 2016, Páginas 145 - 156

A partir de los datos observados en las inspecciones de los edificios, se buscó verificar las mayores incidencias de anomalías y la posibilidad de asociar las manifestaciones patológicas de las fachadas de los edificios con la dirección geográfica.

\section{RESULTADOS Y ANÁLISIS}

En los 52 edificios inspeccionados inicialmente, según procedimientos indicados anteriormente, las principales manifestaciones patológicas observadas están indicadas en la Figura 4:
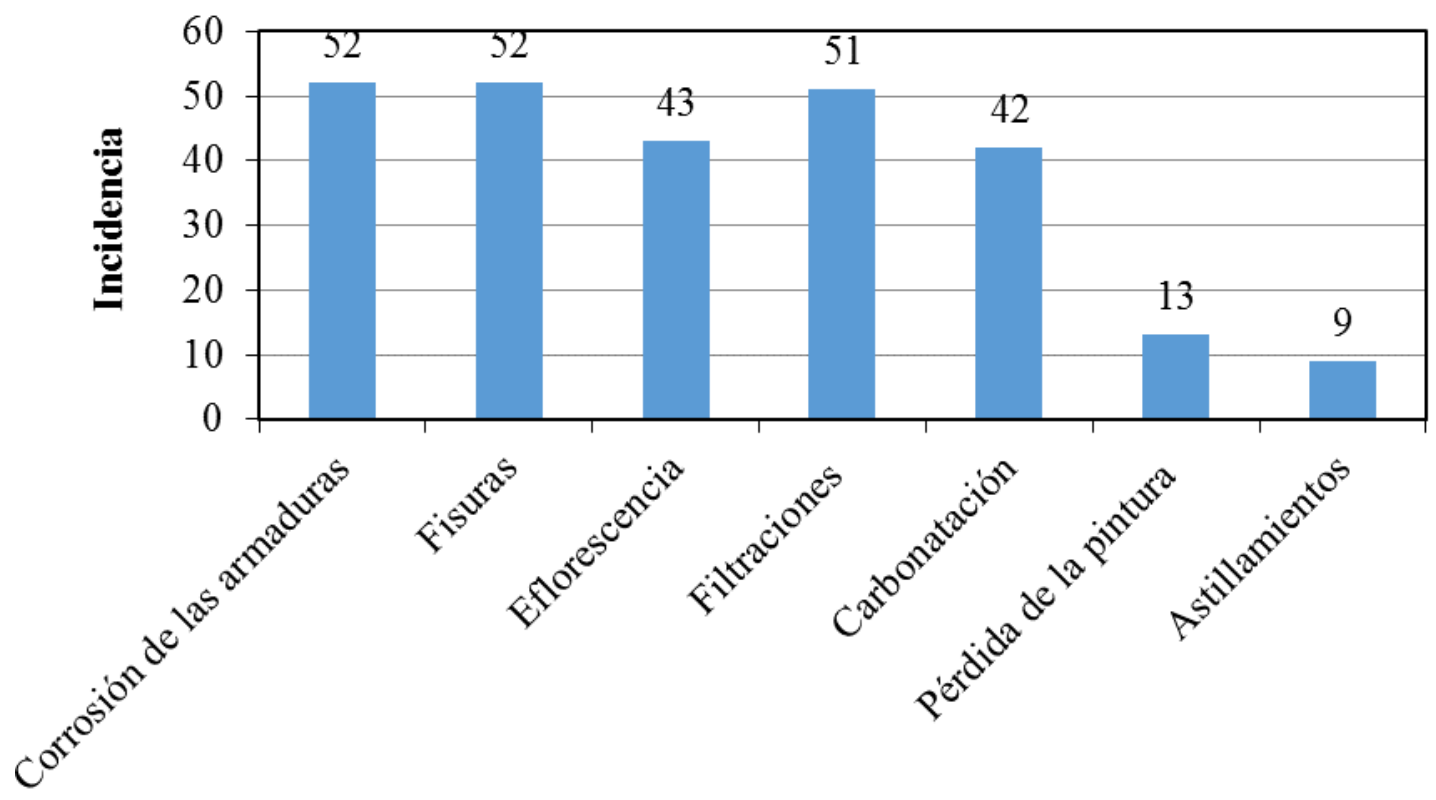

Figura 4. Ocurrencia de manifestaciones patológicas.

Con uma incidencia en 100\% de los condominios inspeccionados, la Corrosión de la Armadura es la manifestación patológica de mayor frecuencia en función de tener vários agentes causantes, como filtraciónes, falta de recubrimento de la armadura, exposición a ambientes confinados con cloruros (tanques de agua), entre otras causas.

La segunda anomalia con mayor incidencia fueron las fisuras, que también es una manifestación que proviene de várias causas como: Dilatación térmica, falta de apoyos, dinteles o vigas, causadas por Corrosión entre otras. Posterior a las fisuras se tienen las filtraciones como la tercera anomalia de mayor incidencia, seguida de Eflorescencia y Carbonatación de las estruturas. De las fallas analizadas la de menor incidencia son aquellas existentes en las fachadas de los edifícios, como pérdida de la pintura, consideradas, de manera general, como Astillamientos.

En la Figura 5 es posible verificar las incidencias de amomalias relacionadas a la Región construtiva de la edificación, y que en este caso se observan anomalias de la fachada en aproximadamente $50 \%$ de las edificaciones evaluadas, indicando que no son apenas los Astillamientos que están relacionados a este problema, conforme puede ser visto a continuación: 
Revista ALCONPAT, Volumen 6, Número 2, Mayo - Agosto 2016, Páginas 145 - 156

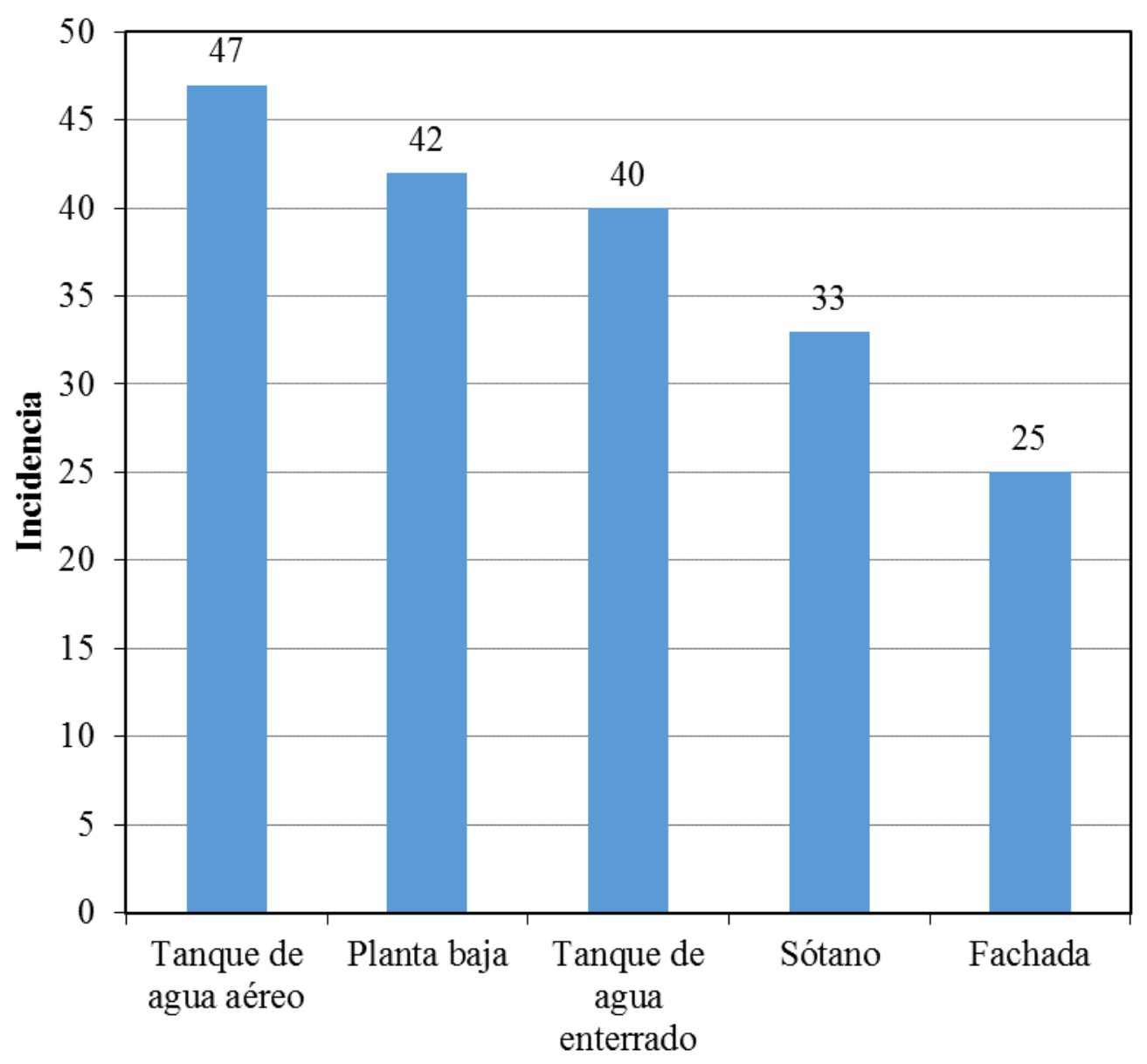

Figura 5. Localización de las manifestaciones patológicas.

Es posible observar en la Figura 5, que las fachadas de los edificios poseen la menor incidencia de manifestaciones patológicas, por tanto, ocurren en $48 \%$ de los edificios inspeccionados, es decir en 25 edificios. A pesar de la menor incidencia, son observadas en las fachadas las manifestaciones patológicas y se presentan en la Tabla 1.

Tabla 1 - Incidencia de manifestaciones patológicas en fachadas.

\begin{tabular}{|l|c|}
\hline Manifestación patológica & Cantidad incidente en la fachada. \\
\hline Fisuras & 25 \\
\hline Eflorescencia & 14 \\
\hline Astillamientos & 22 \\
\hline Filtraciones & 7 \\
\hline
\end{tabular}

Los porcentajes de incidencia de manifestaciones patológicas incidentes en las fachadas de los edificios inspeccionados están indicados en la Tabla 2, divididos de acuerdo con la dirección geográfica en que fueron observados. 
Revista ALCONPAT, Volumen 6, Número 2, Mayo - Agosto 2016, Páginas 145 - 156

Tabla 2 - Porcentajes de incidencia de manifestaciones en cada dirección geográfica.

\begin{tabular}{|l|c|c|c|c|}
\hline \multicolumn{1}{|c|}{ Dirección Geográfica } & Fisuras & Eflorescencia & Astillamientos & Filtraciones \\
\hline Norte & 40,0 & 28,6 & 31,8 & 0,0 \\
\hline Sur & 16,0 & 14,3 & 22,7 & 14,3 \\
\hline Este & 16,0 & 28,6 & 18,2 & 57,1 \\
\hline Oeste & 28,0 & 28,6 & 27,3 & 28,6 \\
\hline
\end{tabular}

En la Tabla 1 es posible observar que en todos los edificios que presentaron manifestaciones patológicas en las fachadas ocurrió la incidencia de fisuras, siendo que la mayor parte de las fisuras, como se indica en la Tabla 2, ocurren en las fachadas que apuntan para la dirección Norte, que, en Curitiba, recibe la mayor incidencia solar, y que puede sufrir una variación de temperatura de hasta $10^{\circ} \mathrm{C}$ en un mismo día, como se indica en la Figura 7. Ejemplos de estas incidencias son mostrados en la Figura 6. Los astillamientos también ocurren, en su mayoría, en las mismas fachadas, una vez que la variación de temperatura, además de fallas en la ejecución, también influyen en este daño.

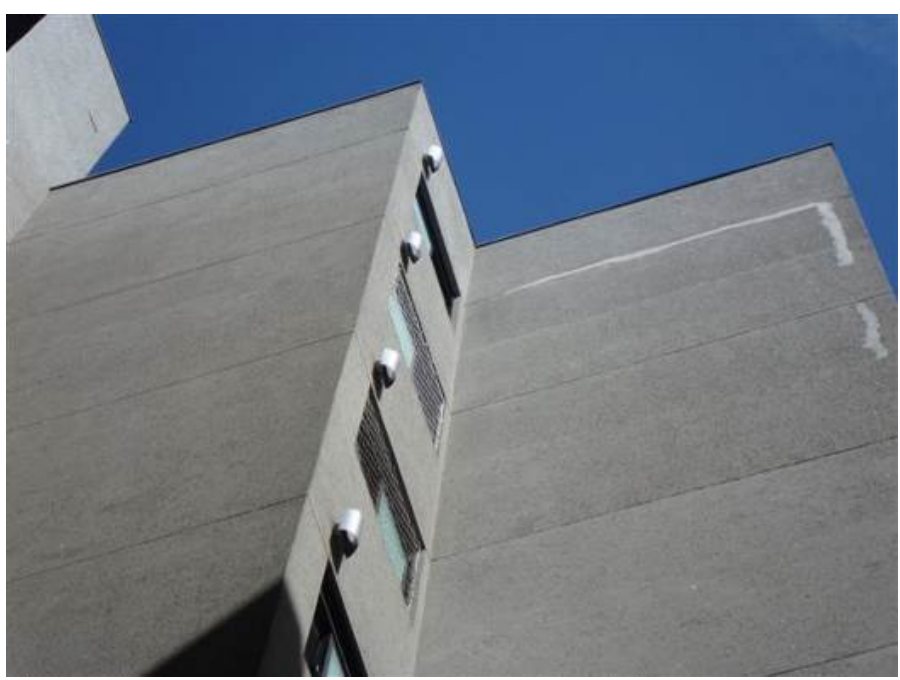

a) Fisuras de variación térmica.

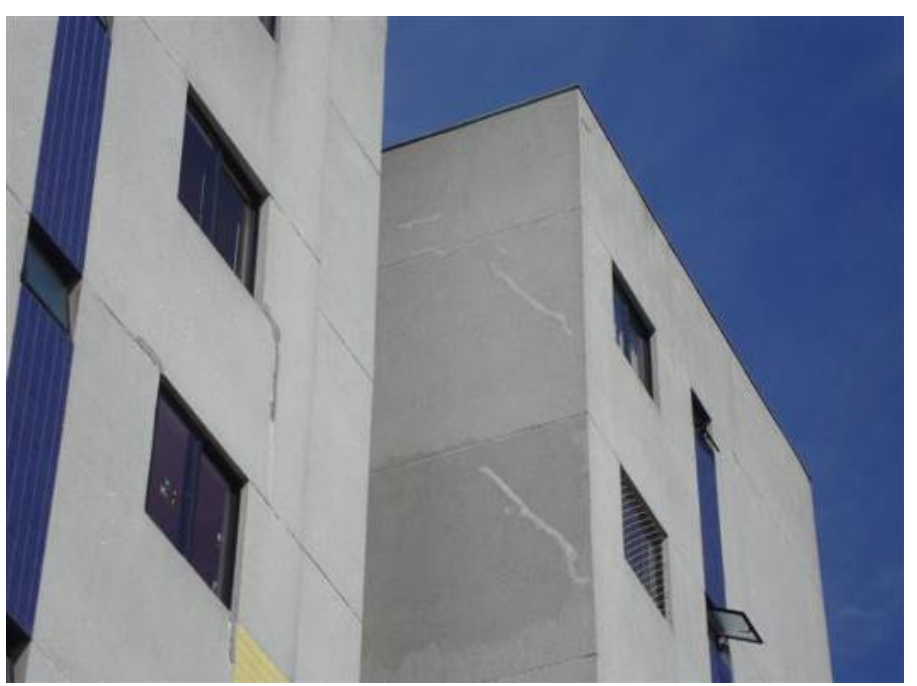

b) Fisuras de movimiento de la estructura.

Figura 6. Fisuras de fachada. 
Revista ALCONPAT, Volumen 6, Número 2, Mayo - Agosto 2016, Páginas 145 - 156

Las Eflorescencias, incidentes en 56\% de los edificios con manifestaciones patológicas en las fachadas, aparecen en menor cantidad en la Región Sur, que posee menor incidencia de vientos y lluvias. En las demás direcciones geográficas el porcentaje de incidencia fue el mismo, no siendo posible establecer una correlación entre esta manifestación y la dirección geográfica.

Ya las Filtraciones, presentes en $28 \%$ de los edificios, aparecen en mayor cantidad en la cara este de las fachadas, dirección con gran incidencia de vientos y lluvias. En la Figura 8 se presenta un mapa de dirección predominante de los vientos en la Región, mostrando la dirección Este con mayor incidencia, conduciendo las lluvias también en esa dirección, justificando la mayor incidencia de Filtraciones en esta Región.

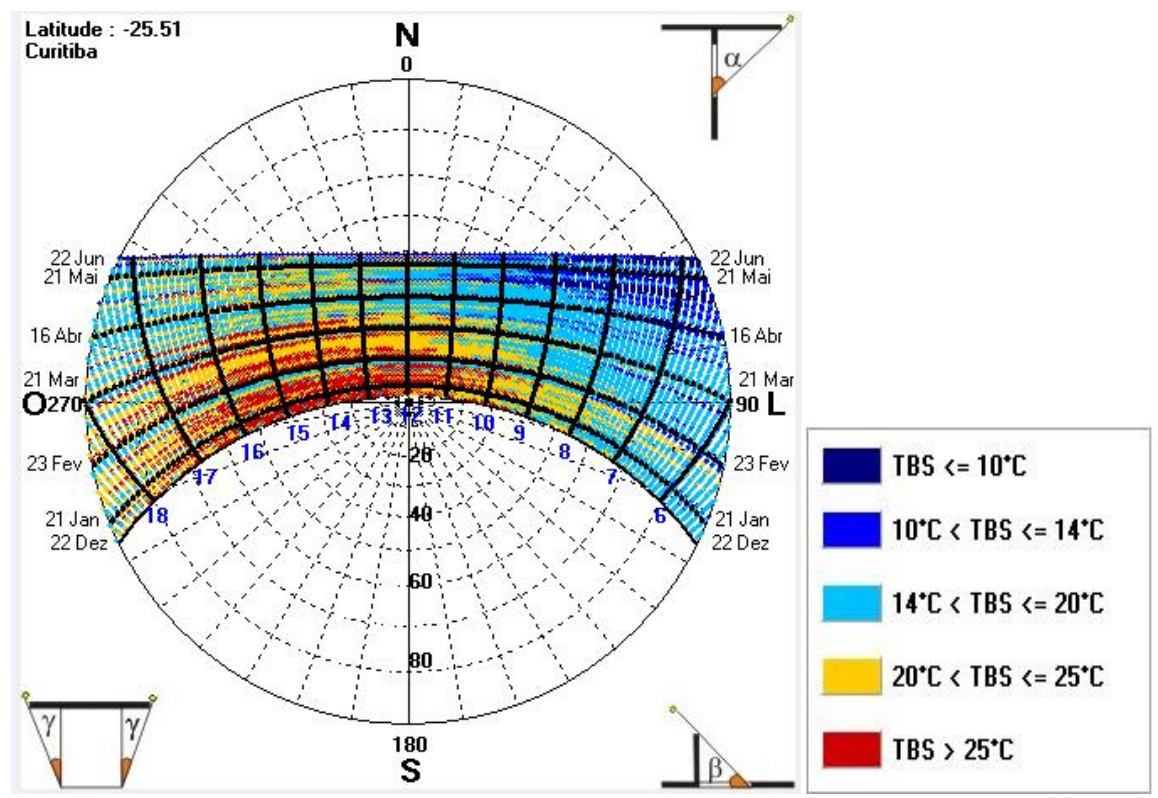

a) Temperaturas de diciembre a junio.

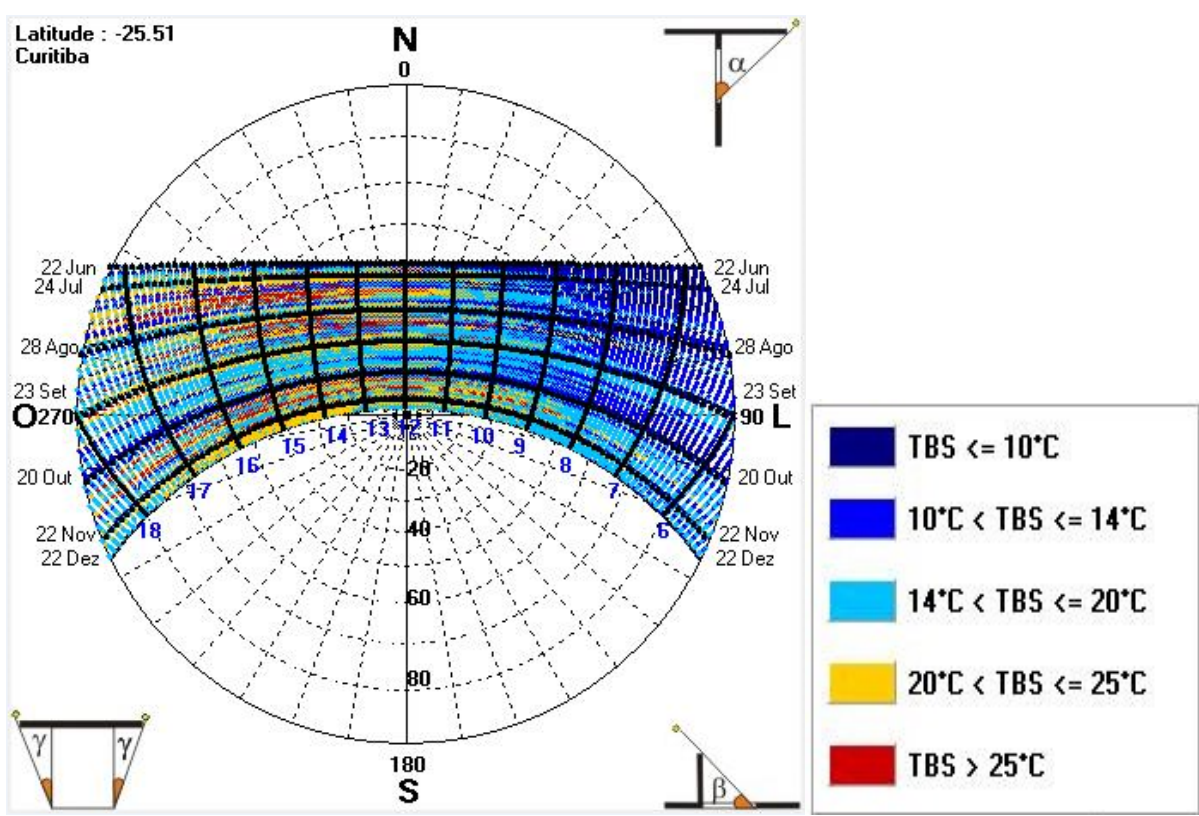

b) Temperaturas de junio a diciembre.

Figura 7. Temperaturas medias en Curitiba. 
Revista ALCONPAT, Volumen 6, Número 2, Mayo - Agosto 2016, Páginas 145 - 156

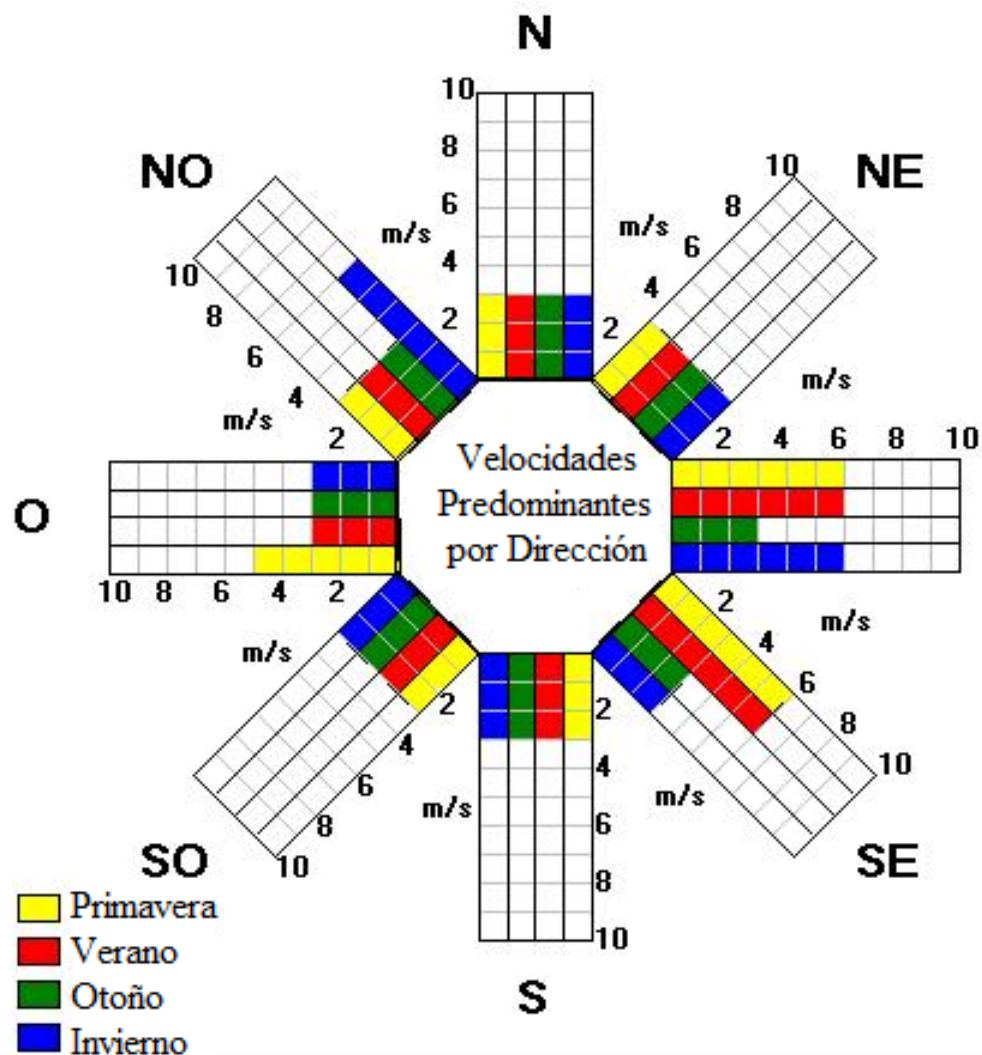

a) Velocidad de los vientos.

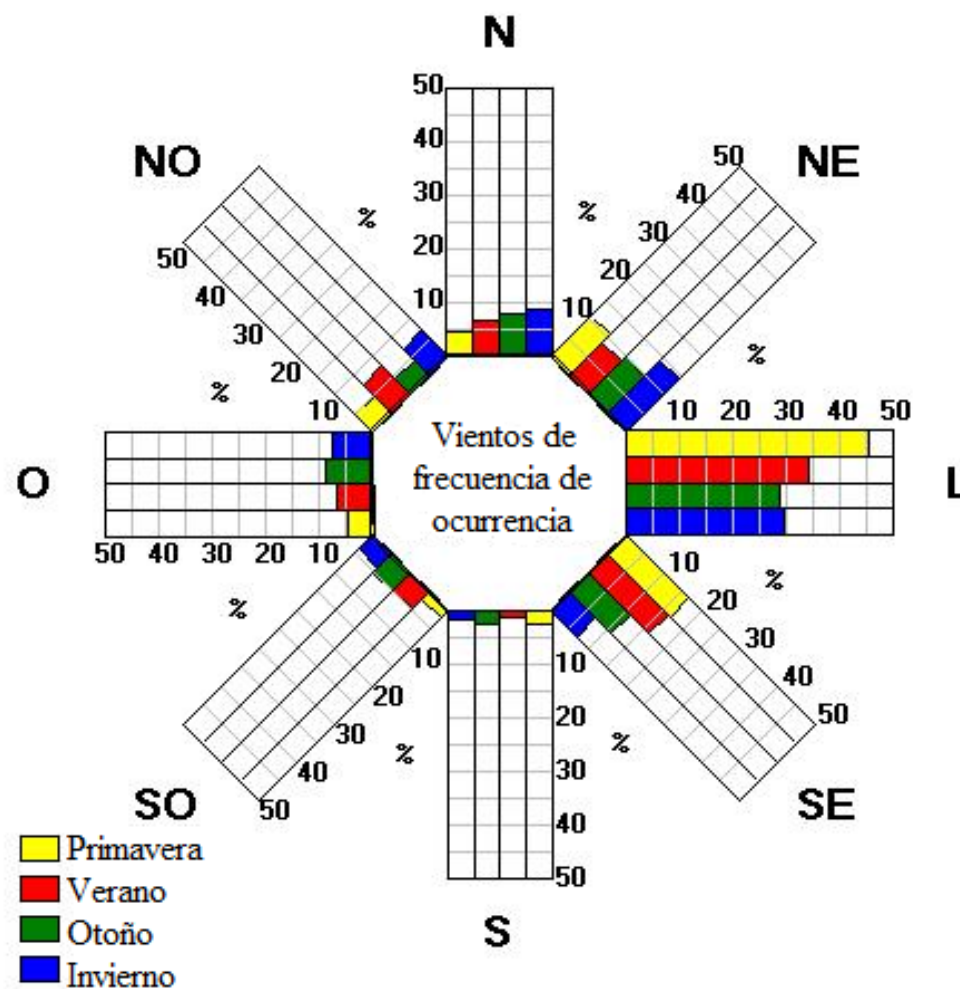

b) Frecuencia de los vientos.

Figura 8. Incidencia de vientos en Curitiba. 
Revista ALCONPAT, Volumen 6, Número 2, Mayo - Agosto 2016, Páginas 145 - 156

En la Figura 7, la numeración de 6 a 18 representa las horas del día en que ocurre la incidencia solar en una fachada apuntando para el Norte, y los datos son algunos días aleatorios escogidos para ser citados en la carta solar. En la Figura 6a, es posible observar, por ejemplo, que la temperatura a las 6:00h de la mañana del día 22 de diciembre aparece entre $14^{\circ} \mathrm{C} \mathrm{y} 20^{\circ} \mathrm{C}$ y a las 13:00h la temperatura es superior a $25^{\circ} \mathrm{C}$. En la Figura 8 son indicadas las velocidades y porcentajes de incidencia de los vientos en las diferentes direcciones geográficas y estaciones Del año.

De las fallas observadas en los edificios se destacan la Corrosión de las armaduras que aparecieron en el 100\% de los edificios analizados y las fisuras en las fachadas que también inciden en 100\% de los edificios que poseen problemas en las fachadas.

\section{CONCLUSIONES}

De los 52 casos analizados, fue observada la existencia de Corrosión de las armaduras y fisuras en todos ellos, además de filtraciones en 98\% de los edificios, Eflorescencia en 83\% y Carbonatación en $81 \%$ como principales manifestaciones patológicas. Con menor incidencia, 25\% y 17\%, aparecen, respectivamente la pérdida de pinturas y astillado en fachadas.

Las manifestaciones patológicas en fachadas aparecen en 25 de los 52 edificios inspeccionados, siendo observadas fisuras en las 25 fachadas, pérdida de pintura o astillamiento en 22 fachadas, Eflorescencia en 14 y Filtraciones en 7 fachadas de edificios.

La dirección geográfica con mayor incidencia solar presentó la mayor cantidad de manifestaciones patológicas, destacándose las fisuras y astillamientos, lo que indica una posible relación entre la esta dirección geográfica y los tipos de daños.

La mayor presencia de Filtraciones ocurre en fachada que apunta para la dirección geográfica con mayor velocidad e incidencia de vientos, indicando una probable relación entre estos factores y la incidencia de Filtraciones.

A pesar de los indicios existentes entre las direcciones geográficas y la incidencia de manifestaciones patológicas, es necesario considerar también los factores ambientales como incidencia solar, variaciones de temperatura y direcciones predominantes de vientos y lluvias para establecer correlaciones entre dirección geográfica y manifestaciones patológicas.

\section{AGRADECIMIENTOS}

Los autores agradecen a la empresa Estruktor Patologia das Construcciones Ltda por efectuar las inspecciones y ceder los datos para la elaboración de este estudio.

\section{REFERENCIAS}

Abreu, D. W. M., Lins, A. M. T., Silva, M. S., Farias, M. B. S. (2013), "Patologias no revestimiento de fachadas dos edifícios históricos da ciudad de Campina Grande”, In: 5. EnPAC, Campina Grande (PB).

American Society for Testing and Materials. ASTM - E 632-81. "Standard recommended practice for developing short-term accelerated test for prediction of the service life of building components and materials”, Philadelphia, 1996.

Antunes, G. R. (2010), "Estudo de manifestaciones patológicas de fachadas em Brasíla Sistematização da incidencia de casos”, Dissertação de mestrado. UNB.

Barros, M. M. S. B.; Sabbatini, F. H. (2001), "Produção de revestimientos cerâmicos para paredes de vedação em alvenaria: Diretrizes básicas”, (São Paulo, Brasil: EPUSP).

Evaluación de manifestaciones patológicas en edificios en función de la orientación... 
Revista ALCONPAT, Volumen 6, Número 2, Mayo - Agosto 2016, Páginas 145 - 156

Galletto, A., Andrello, J. M. (2013), “Patologia em fachadas com revestimientos cerâmicos”, In: CINPAR - IX Congresso Internacional sobre Patología y Recuperación de Estruturas, João Pessoa (Brasil).

Japiassú, J., Carasek, H., Cascudo, O., Velosa, A. L. (2014), "Caracterização da interface azulejo/argamassa de fachadas históricas”, Revista ALCONPAT, V. 4, No. 1, pp. 55-76.

Lembo, F. (2011), "Pathologies of industrialized bulding made by bearing precast concrete panels. The case study of intervention with the same building system at Chiaromonte, Francavilla in Sinni and Lagonegro", In: XII DBMC - International Conference on durability of Building Materials and Components, Porto (Portugal).

Melo, C. M., Carasek, H. (2014), "Relationship between the deterioration of multi story building facades and driving rain”, Revista de la Construción, V. 13, No. 1, pp. 64-73.

Resende, M. M.; Barros, M. M. S. B.; Campos, J. S. (2001), “A influencia da manutenção na durabilidade dos revestimientos de fachada de edifícios”, In: Workdur - II Workshop sobre durabilidade das construcciones, São José dos Campos (Brasil), pp. 144-154.

Romero, N., Dupuy, C., Quiñones, J. (2011), "Influencia de la contaminación atmosférica em la fachada de rascacielos, caso Torre Colpatria”, Revista ALCONPAT, V. 1, No. 3, pp. 228-235.

Shirakawa, M. A. (1995), "Identificação de fungos em revestimientos de argamassa com bolor evidente”, In: I Simpósio Brasileiro de Tecnologia das Argamassas, Goiânia (Brasil), pp.402-10. 